\title{
THE SEQUENCE EQUIVALENCE PROBLEM FOR \\ DOL SYSTEMS IS DECIDABLE *
}

K. CULIK II

University of Naterloo, Water 700 , Ont., Canada

\section{FRI ̌̌}

University of New England, Armidale, N.S.W., Australia

\section{Abstract}

The sequence equivalence problem for DOL systems is shown to be decidable. In an algebraic formulation the sequence equivalence problem for DOL systems can be stated as follows: Given homomorphisms $h_{1}$ and $h_{2}$ on a free monoid $\Sigma^{*}$ and a word $\sigma$ from $\Sigma^{*}$, is $h_{1}^{n}(\sigma)=h_{2}^{n}(\sigma)$ for all $n>0$ ?

\section{Introduction}

Given two homomorphisms $h_{1}$ and $h_{2}$ on a free monoid $\Sigma^{*}$ and a word $\sigma$ in $\Sigma^{*}$, can we determine whether $h_{1}^{n}(\sigma)=h_{2}^{n}(\sigma)$ for all $n>0$ ? This is an algebraic formulation of the well-known DoL sequence equivalence problem. The problem originated in Lindenmayer systems which are mathematical models of cellular development. In that context it can be restated as the problem of the developmental equivalence of two genetic encodings in filamental organisms developing deterministically without interaction. Lindenmayer systems without interaction (DOL systems) were introduced in [5] and the equivalence problem for them was posed shortly afterwards in [8].

Here we summarize [1] and [2] which together show that this problem is decidable. The aim of the present paper is to give an intuitive overview to the proof techniques used in [1] and [2]. We give only outines of most proofs, readers interested in the technical details are referred to the two above papers. However, it may be useful to read this paper first since the informal outlines of some of the proofs will make the reading of [1] and [2] easier.

* This paper summarizes two previous works supported by the ilational Research Council of Canada, Grant No. A7403 and was written when the first author was visiting the University of Karlsruhe, Germany. 
First we explain intuitively the basic ideas of our solution of the DoL equivalence problem. Henceforth, by equivalence we mean sequence equivalence. The technical terms which are used in the introduction but defined only later are enclosed in quotation marks on firt use.

We start by showing that, without loss of generality, the testing for equivalence may be restricted to "normal" systems. Then we introduce an essential auxiliary notion, namely, the "balance" of a string $w$ in $\Sigma^{*}$ with respect to two homomorphisms $h_{1}, h_{2}$ on $\Sigma^{*}$ (or with respect to two DOL system based on these homomorphisms). The balance of $w$ is the difference of the length of $h_{1}(w)$ and $h_{2}(w)$. The central intermediate result is Theorem 4 showing that there exists a fixed bound on the balance of all prefixes of a 11 strings generated by two equivalent normal DoL systems. In section 3 and 4 this result is proved. First "simple" systems are considered. A normal system is simple iff it has no "subsystem" in the sense of general algebra. If a system has a subsystem, then the underlying set of the subsystem is called a "subalphabet". For simple systems it is shown first, using the methods of 1 inear algebra, that the balance of every "long" prefix is "very small" compared to its length. Then, using combinatorial methods, this result is strengthened to show that the balance of two equivalent systems is bounded if at least one of the systems is simple.

For two equivalent systems neither of which is simple we find a common subalphabet and show that either

1) all substrings of the language generated by the systems which are entirely in this subalphabet are "short" (such a subalphabet is called "limited") or

2) that every two systems forming a pair "induced" by this subalphabet are equivalent. Another pair of normal systems is obtained by "removing" the subsystem (i.e. by omitting the symbols from the common subalphabet). As before, these "remainder"-systems are equivalent because the original systems are equivalent. Since both the subsystem and the remainder system are systems over a smaller alphabet we can use the boundedness as an induction hypothesis. The base of the induction deals (essentially) with systems over one letter so the claim is easy to verify. This allows us to assume that the remainder-pair and (in the case of a subalphabet which is not 1 imited) also every induced-pair have bounded balance. As the case of limited subalphabets causes no problem, this allows us to construct a bound on the balance for the original pair. One of the more important technical details which has been omitted above is the following. In every step of the induction we have to consider the non-propagating systems and another singular case separately. Since a propagating system may have a non-propagating remainder system we cannot include the propagating property into the requirements for normality.

In the last section we design a decision procedure which works for every family of DOL systems for which equivalence implies bounded balance. However, we have shown that 
we need, without loss of generality, only to aive the decision procedure for normal systems. Therefore it is sufficient that equivalence of normal systems implies bounded balance, this in fact we show in Section 5 .

The normal systems are used for technical rather than essential reasons and we conjecture that every pair of equivalent DOL systems has bounded balance. It also seems feasible to sharpen the proof of Theorem 3 so that it would give a bound on the balance of a pair of DOL systems rather then to show only that such bound exists. This will then allow the complexity of the whole procedure to be determined.

\section{Notation}

Given an alphabet $\Sigma, \Sigma^{*}$ denotes the free monoid generated by $\Sigma$, with unit (empty string) $\varepsilon$. A DOL system is a 3 -tuple $G=(\Sigma, h, \sigma)$ consisting of alphabet $\Sigma$, homomorphism $h$ and a starting string $\sigma \in \Sigma^{*} . L(G)$, the language generated by $G$, is defined as $\left\{h^{n}(\sigma): n \geq 0\right\}$. $G$ is said to be reduced, if every symbol from $\Sigma$ occurs in at least one $h^{n}(\sigma), n \geq 0$. To reduce $G$ means to omit from $\Sigma$ all the symbols which do not have this property.

For $w \in \Sigma^{*}$ and $a \in \Sigma, \#_{a} w$ denotes the number of occurrences of $a$ in $w$. If $\left(a_{1}, \ldots, a_{n}\right)$ is an ordering of $\Sigma$, then $\left(\#_{a_{1}} w, \ldots, \#_{a_{n}} w\right.$ ) is called the Parikh vector of $w$ and is denoted by $[w]$. The matrix $M=\left(m_{i j}\right)_{\substack{1 \leq i \leq n \\ 1 \leq j \leq n}}$, where $m_{i j}=\#_{a_{j}} h\left(a_{i}\right)$ is called the growth matrix for $G$.

If $i$ is a number, $|i|$ denotes the absolute value of $i$; if $w$ is a string $|w|$ denotes the length of $w$; later on $|A|$ is also used for length of a vector $A$ or maximum characteristic value of a matrix $A$. For $w \in \Sigma^{*}$, let $\min (w)=\left\{a: \#_{a} w>0\right\}$. Given $G=(\Sigma, h, \sigma)$, we say that $w$ is a $G$-prefix (G-substring, $G$-suffix) if $w$ is a prefix (substring, suffix) of $h^{n}(\sigma)$ for some $n \geq 0$. Two DOL systems $G_{i}=\left(\Sigma, h_{j}, \sigma_{i}\right), i=1,2$ are called (sequence) equivalent if $h_{1}^{n}\left(\sigma_{1}\right)=h_{2}^{n}\left(\sigma_{2}\right)$ for all $n=0,1, \ldots$. Two DOLsystems $G_{1}, G_{2}$ are called Parikh equivalent if $\left[h_{1}^{n}\left(\sigma_{1}\right)\right]=\left[h_{2}^{n}\left(\sigma_{2}\right)\right]$ for all $n=0,1, \ldots$. The balance (with respect to $G_{1}, G_{2}$ ) of a string $w$ in $\Sigma^{*}$ is defined by $B(w)=|| h_{1}(w)|-| h_{2}(w) \|$. If there exists $c \geq 0$ so that $\beta(x) \leq c$ for all $G_{1}$-prefixes $x$, then the pair $\left(G_{1}, G_{2}\right)$ is said to have bounded balonce. In this case the smallest such $c$ is called the bazance of the pair $\left(G_{1}, G_{2}\right)$.

For two sets $A, B, A \cup B$ denotes their union. If $A, B$ are disjoint, we stress this by writing $A+B$ for the union. Finally, we will often write a instead of $\{a\}$ for a oneelement set. 


\section{The Normal Systems}

Let $G=(\Sigma, h, \sigma)$ be a $D O L$ system. We define the function $m: P(\Sigma) \rightarrow P(\Sigma)$, where $P(\Sigma)$ is the set of all subsets of $\Sigma$ by putting

$$
\begin{aligned}
& m(\phi)=\phi, \\
& m(\{a\})=\min (h(a)) \text { for } a \in \Sigma, \\
& m(A \cup B)=m(A) \cup m(B) .
\end{aligned}
$$

It is easy to see that $m^{i}(a)=\min \left(h^{j}(a)\right)$ for all $i \geq 1$. We will write $m(a)$ for $m(\{a\})$ and use $m_{1}, m_{2}, m_{12}$ etc. to denote similar functions based on $h_{1}, h_{2}, h_{1} h_{2}$, etc.

Definition 1 A DOL system $G=(\Sigma, h, \sigma)$ is called an 2 -system if $\Sigma=\Sigma_{z}+\Sigma_{c}+\Sigma_{r}$ is a decomposition of $\Sigma$ into three non-empty disjoint sets such that $h(a) \in \Sigma_{\eta} \Sigma_{c}^{*}$ for $a \in \Sigma_{l}, h(a) \in \Sigma_{c}^{*}$ for $a \in \Sigma_{c}, h(a) \in \Sigma_{c}^{*} \Sigma_{r}$ for $a \in \Sigma_{r}$, and $\sigma \in \Sigma_{\Sigma^{2}} \Sigma_{c}^{*} \Sigma_{r}$. We call $\Sigma_{c}$ the core of $\Sigma, \Sigma_{\eta}$ is called the teft side, $\Sigma_{r}$ the might side of $\Sigma$. The number of symbols in the core $\Sigma_{C}$ of $\Sigma$ is called the order of $G$.

Definition 2 A DOL system $G=(\Sigma, h, \sigma)$ is called normal if

$G$ is an $2 r$-system,

$G$ is reduced,

holds for every $a, b \in \Sigma_{c}$.

Now, we show that, without loss of generality, the testing for equivalence can be restricted to the normal systems.

Theorem 1 The sequence equivalence problem is decidable for DOL systems iff it is decidable for normal systems.

Proof Outline (for details see [2] Lemma 1,2 and Theorem 1). Given a DOL system $G=(\Sigma, h, \sigma)$, let $G^{j, k}=\left(\Sigma, h^{j}, h^{k}(\sigma)\right)$ be the $(j, k)$ speed-up of $G$. It is easy to see that two DOL systems $G_{1}$ and $G_{2}$ are equivalent iff all the $j$ pairs of DOL systems $G_{1}^{j, k}, G_{2}^{j, k}$ for $k=0, \ldots, j-1$ are equivalent.

Given any pair $G_{j}=\left(\Sigma, h_{j}, \sigma\right) i=1,2$ of DOL systems we can effectively find $j$ so that the speed-up systems $G_{i}^{j, k}$ are normal for $i=1,2$ and $k=0, \ldots, \ldots, j-1$. We first find $h_{1}, h_{2}$ for which $h_{1}{ }_{1}$ and $h_{2}^{k_{2}}$ meet condition (3) of normality. Then we choose $j$ as the least common multiple of $h_{1}$ and $h_{2}$.

Finally, we reduce the systems $G_{i}^{j, k}$ for $i=1,2$ and $k=0, \ldots, j-1$, and if the systems are not 2 m-systems we create the sides "artificially". 


\section{The $2 r-S i m p l e ~ S y s t e m s$}

Definition 3 Let $G=\left\langle\Sigma_{Z}+\Sigma_{c}+\Sigma_{r}, h, \sigma\right\rangle$ be an 2 r-system. A homomorphism $h$ is called zx-simple if for every $a, b \in \Sigma_{c}$ and every $k>0$ there is $j>0$ such that $a \in m k j(b)$. Equivalently, calling $h z_{r}$-imeducibze if for every $a, b \in \Sigma_{c}$ there is $j>0$ such that a $\in \mathrm{m}^{j}(b), h$ is 2 rusimple iff $h^{k}$ is 2 -irreducible for all $k \geq 1$. We call $G 2 r$-simple if $h$ is zr-simple.

If $G$ is $2 r$-simple and normal, then from $a \in m^{k j}(b)$ we get $a \in m(b)$.

Putting $a=b$ we get $a \in m(a)$, which implies in turn that $a \in m^{k}(b)$ for all $i \geq 1$. Thus if $G$ is normal, $G$ is $z r$-simple iff $m(b)=\Sigma_{c}$ for all $b \in \Sigma_{c}$. However, the following lemma is needed for systems not necessarily normal.

Lemma 1 Let $G_{j}=\left(\Sigma, h_{j}, \sigma\right), i=1,2$ be two Pariskh equivalent DoL systems, $G_{1} 2 r-s i m p l e$ and of order at least two $(|\Sigma| \geq 2)$. Then for every $\varepsilon>0$ there is $n_{0}>0$ such that for every $w \in \Sigma^{*}, w \notin\left(\Sigma_{I} \cup \Sigma_{r}\right)^{*}, \beta\left(h_{1}^{n}(w)\right) \leq \varepsilon\left|h_{1}^{n}(w)\right|$ for all $n>n_{0}$.

Proof Outline (for details see [2], Lemma 3). By [7], for $i=1,2$, the Parikh vector $v_{i}^{k}$ of $h_{i}^{k}(\sigma)$ can be written as $v_{i}=x M_{i}^{k}$ where $x$ is the Parikh vector of $w$ and $M_{i}$ is the growth matrix of $M_{j}$. Therefore the computation of $v_{j}^{1}, v_{i}^{2}, \ldots$ constitutes the power method for computing the characteristic $u_{i}$ vector of $M_{i}$ corresponding to the largest eigenvalue of $M_{1}$. From the observation that $\beta\left(u_{1}\right)=B\left(u_{2}\right)=0$ then easily follows that

$$
\lim _{n \rightarrow \infty} \frac{\beta\left(h_{1}^{n}(w)\right)}{\left|h_{1}^{n}(w)\right|}=0 .
$$

$\square$

Theorem 2 Let $G_{i}=\left(\Sigma, h_{i}, \sigma\right), i=1,2$, be two Parikh equivalent DOL systems, $G_{1}$ I. $r$ simple and $|\Sigma| \geq 2$. Then for every $\varepsilon>0$ there is $K>0$ such that for every $G_{I}$-prefix $w,|w|>K$ we have $\beta(w) \leq \varepsilon|w|$.

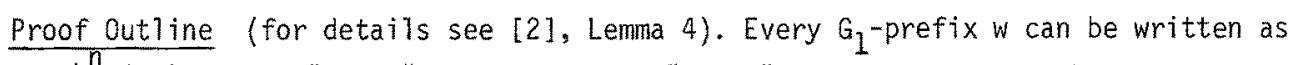
$w=h_{1}^{n} o\left(u_{1}\right) x$ for a "large" integer $n_{0}$ and "short" string $x \in \Sigma^{*}$. We have $\beta(w) \leq \beta\left(n_{1}\left(u_{1}\right)\right)+\beta(x)$. Given $\varepsilon>0$ we have $\beta\left(n_{1}^{n_{0}}\left(u_{1}\right)\right) \leq \varepsilon / 2\left|u_{1}\right| \leq \varepsilon / 2|w|$ by Lemma 1 ana $\beta(x) \leq \varepsilon / 2|W|$ since $x$ is snort.

We have shown that the balance of "long" strings is small with respect to their length. However, our goal is to show a stronger result, namely, that the balance is bounded. Before we can show this we will need a few auxiliary notions.

The notion of a derivation forest of a string $w$ with respect to a $D O L$ system $G$ is obtained by the obvious modification of the well-known notion of a derivation tree for a context-free grammar. The difference is that in DOL systems we have an axiom (string of symbols) rather than a single starting symbol of context-free grammars. 
Let $F$ be the derivation forest of $h^{n}(\sigma)$ for some $n>0$ with respect to DOL system $G=(\Sigma, h, \sigma)$. A path in $F$ from a node on the lowest level (of $\sigma$ ) to a node $x$ on level $n$ is called the choin of $\alpha$. Formally, a chain $q$ is a string in $(\Sigma \times N)$ such that if $q=\left(a_{0}, k_{0}\right) \ldots\left(a_{n}, k_{n}\right)$, then

$$
1 \leq k_{0} \leq|\sigma|
$$$$
I \leq k_{i} \leq\left|h\left(a_{i-1}\right)\right| \text { for } I \leq i \leq n \text {; }
$$

I.e., $k_{0}$ is the position of $a_{0}$ in $\sigma$, and for $i \geq 1, k_{j}$ determines which of possibly several branches is taken. First components of pairs are clearly redundant but they allow us to state easily the condition (iii).

The string $a_{0} \ldots a_{n}$ is called the trace of chain $q$. A chain $q$ is said to be periodic with period $p$ and prefix (initial segment) $q_{1}$ if $q=q_{1} p q_{2}$ for some $m \geq 2$ and $q_{2}$ is a prefix of $p$. Chain $q$ is leftmost (mightmost) on level if $k_{i}=1\left(k_{i}=\left|h\left(a_{i-1}\right)\right|\right)$. Chain $q$ is fuzly leftmost (fulzy rightmost) if it is leftmost (rightmost) on all levels. For a node $\alpha$ of derivation forest $F$ and a specific occurrence of G-substring $w$, we say that $w$ contains $a$ if $\alpha$ is one of the nodes labeled by a symbol from $w$.

Let $q$ be a periodic chain with prefix $r$ and period $p$. Then there are cyclically repeating (after each $|p| s t e p s$ ) common G-substrings on at least one side of $q$ (see [4], Theorem 11.3 and 11.4).

Theorem 3 Let $G_{j}=\left(\Sigma, h_{j}, \sigma\right)$ for $i=1,2$ be two equivalent $2 r$-systems. Let $G_{1}$ be $2 r-$ simple and of order at least two $(|\Sigma| \geq 2)$. Then the pair $\left(G_{1}, G_{2}\right)$ has bounded balance.

Proof Outline (for details see [1], Lemma 3.1, Theorem 3.2 and [2], Theorem 2. Assume that the pair $\left(G_{1}, G_{2}\right)$ does not have bounded balance (Assumption 1). Therefore, for every $n_{0}>0$ there must exist $n, n \geq n_{0}$, and $u, v$ in $\Sigma^{*}$ so that $n_{1}^{n}(\sigma)=u v$ and the following conditions hold:

$$
\begin{aligned}
& \beta(u)>\beta\left(w_{1}\right) \text { for any prefix } w_{1} \text { of } n_{1}^{j}(\sigma) \text { where } 0 \leq j<n . \\
& \beta(u) \geq \beta\left(w_{2}\right) \text { for any prefix } w_{2} \text { of } n_{1}^{n}(\sigma) . \\
& \beta(u)>\beta\left(w_{3}\right) \text { for any prefix } w_{3} \text { of } u .
\end{aligned}
$$

Let $F$ be the derivation forest of $G_{1}$ and $\alpha$ be the node in $F$ at the last symbol of prefix $u$ at level $n$. Let $q$ be the chain of $\alpha$ in $F$ and let $\alpha_{1}$ and $\alpha_{2}$ be the first two nodes of chain $q$ (from the top) such that the label of $\alpha_{1}$ is the same as the label of $\alpha_{2}$, the label of the left neighbor of $\alpha_{1}$ is the same as that of the left neighbor of $\alpha_{2}$ and the same holds for the right neighbors. Let these common labels be $a, b, c$ from left to right; they, of course, are not necessarily different.

Let the levels of $\alpha_{1}$ and $\alpha_{2}$ in the derivation forest of $G_{1}$ (from top) be $r$ and $r+t$, respectively. Clearly, since $G_{1}$ is 2 rmsimple, there exists a constant $C$ independent 
of $n_{0}$ so that $r+t \leq c$. Note that we have the constant $c_{1}=|\Sigma|+1$ with the property that on levels higher than $C_{1}$ each node of any chain has at least one neighbor both on the left and on the right. This is so since otherwise q would have a fully leftmost (rightmost) initial segment with some symbol occurring at least twice in its trace; therefore, $u$ would be a prefix ( $v$ would be a suffix) of $h_{1}^{j}(\sigma)$ for some $j<n$, which would be in contradiction with condition (i) implied by Assumption 1.

Let $q=q_{1} q_{2} q_{3}$ where $q_{2}$ is the section of $q$ between nodes $\alpha_{1}$ and $\alpha_{2}$. Let $q$ ' be the periodical chain defined by $q^{\prime}=q_{1} q_{2}^{j} q_{4}$ where $j>0$ and $q_{4}$ is a proper prefix of $q_{2}$, and $j$ and $q_{4}$ chosen so that the length of $q^{\prime}$ is the same as the length of $q$. Informally, we have chosen $q^{i}$ so that it coincides with $q$ up to the second occurrence of $a b c$ and then continues periodically. Therefore, there are cyclically repeating longer and longer substrings on both sides of $q^{\prime}$. Specifically $h_{1}^{n-t-r}(a b c)$ is a common substring of $h_{1}^{n-t}(\sigma)$ and $h_{1}^{n}(\sigma)$ which on level $n$ contains node a since chain q goes through node $\alpha_{2}$. Moreover, node $\alpha$ is not close to either end of the common substring since $\alpha_{2}$ is labeled by the middTe symbol $b$ in abc and both $\left|h_{1}^{m}(a)\right|$ and $\left|h_{1}^{m}(c)\right|$ are exponentially growing (for growing $m$ ) by Lemma 3.1.

Now, let $h_{1}^{n-t}(\sigma)=u_{1} x y v_{1}$ and $h_{2}^{n}(\sigma)=u_{2} x y v_{2}$ where $x y=h_{1}^{n-r-t}(a b c), u_{2} x=u$ and $y v_{2}=v$, i.e. the node $\alpha$ on level $n$ is at the last symbol of $x$. Let $u_{1} x=u^{\prime}$. So we have the situation illustrated in Fig. 1.

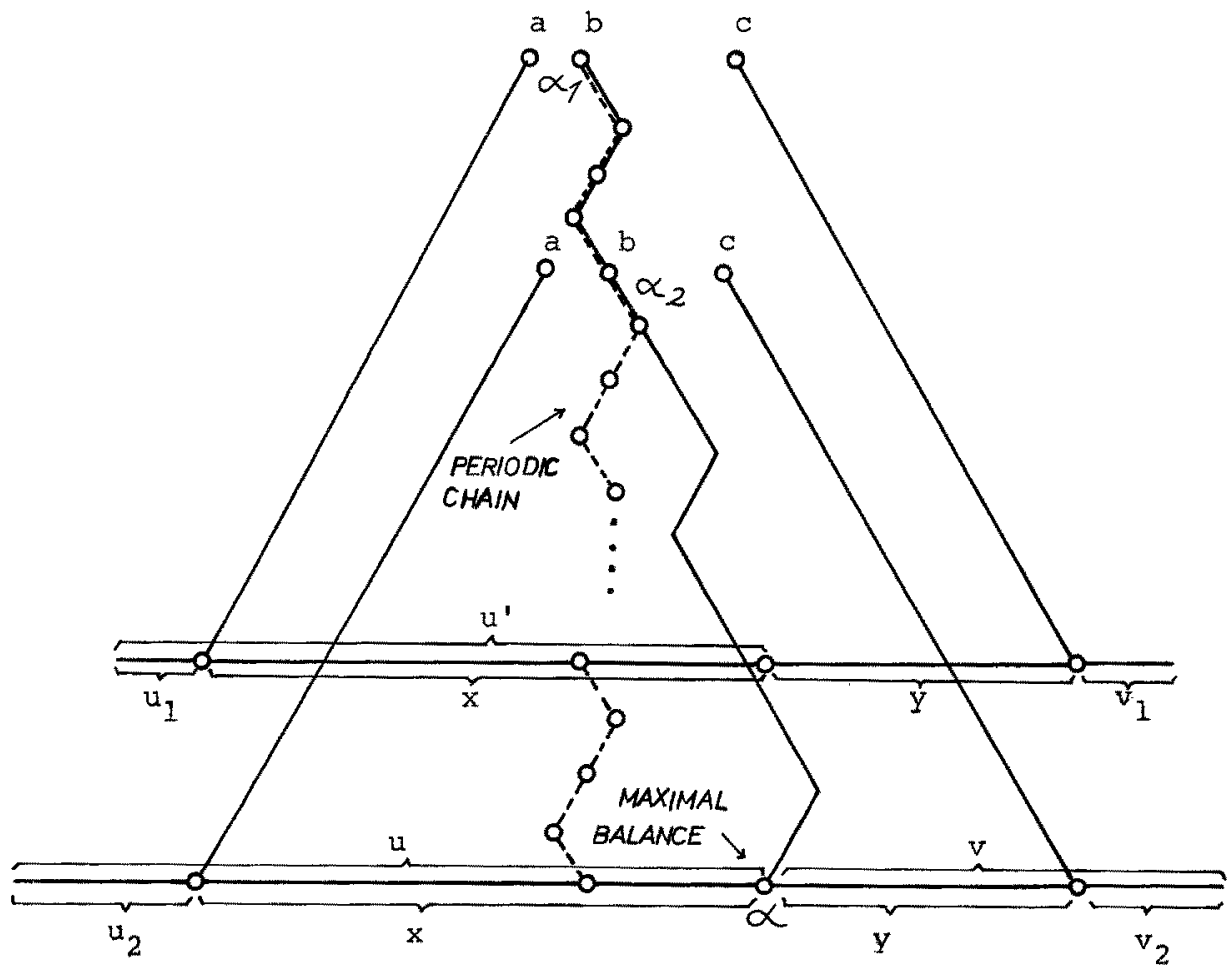

Fig.1 
As the growth of all symbols in a simple system is exponential of the same power, it it easy to see that the length of both $x$ and $y$ is linearly proportional to the length of the whole string $h_{1}^{n}(\sigma)$. Therefore it follows, using Theorem 2 , that for each $\varepsilon>0$ there exists $n_{0}$ so that $\beta(u) \leq \varepsilon|x|$ and $\beta(u) \leq \varepsilon|y|$ where $u, x$, and $y$ are determined by $n_{0}$.

Now, both $h_{1}^{n}(\sigma)$ and $h_{1}^{n-t}(\sigma)$ have $x y$ as a substring with node $\propto$ at the last symbol preceding $y$ on level $n$. Since the two systems are equivalent both $h_{1}(y)$ and $h_{2}(y)$ are substrings of $h_{1}^{n+1}(\sigma)$ and of $h_{1}^{n-t+1}(\sigma)$. We recall that both $\beta\left(u^{\prime}\right)$ and $\beta(u)$ are "very sma11" with respect to $h_{1}(y)$. Assumption 1 gives $\beta\left(u^{\prime}\right)<\beta(u)$, therefore the relative position of $h_{1}(y)$ and $h_{2}(y)$ as substrings of $h_{1}^{n-1}(\sigma)$ differs by a "small" shift $w$ (see Fig. 2) (small with respect to the length of $h_{1}^{n+1}(\sigma)$ and also of $h_{1}(y)$ ), however, the relative position of corresponding strings considered as substrings of $h^{n-t+1}(\sigma)$ differs by even smaller shift $w^{\prime}$. Consider now the "shifts" $z$ and $z$ ' of the longer substrings $h_{1}(x y)$ and $h_{2}(x y)$ on levels $n+1$ and $n-t+1$. Cleariy, $|z|-\left|z^{\prime}\right|=|w|-\left|w^{3}\right|$ and thus also $|z|>\left|z^{\prime}\right|$. Similar results are obtained also where the "shifts" are in opposite directions.
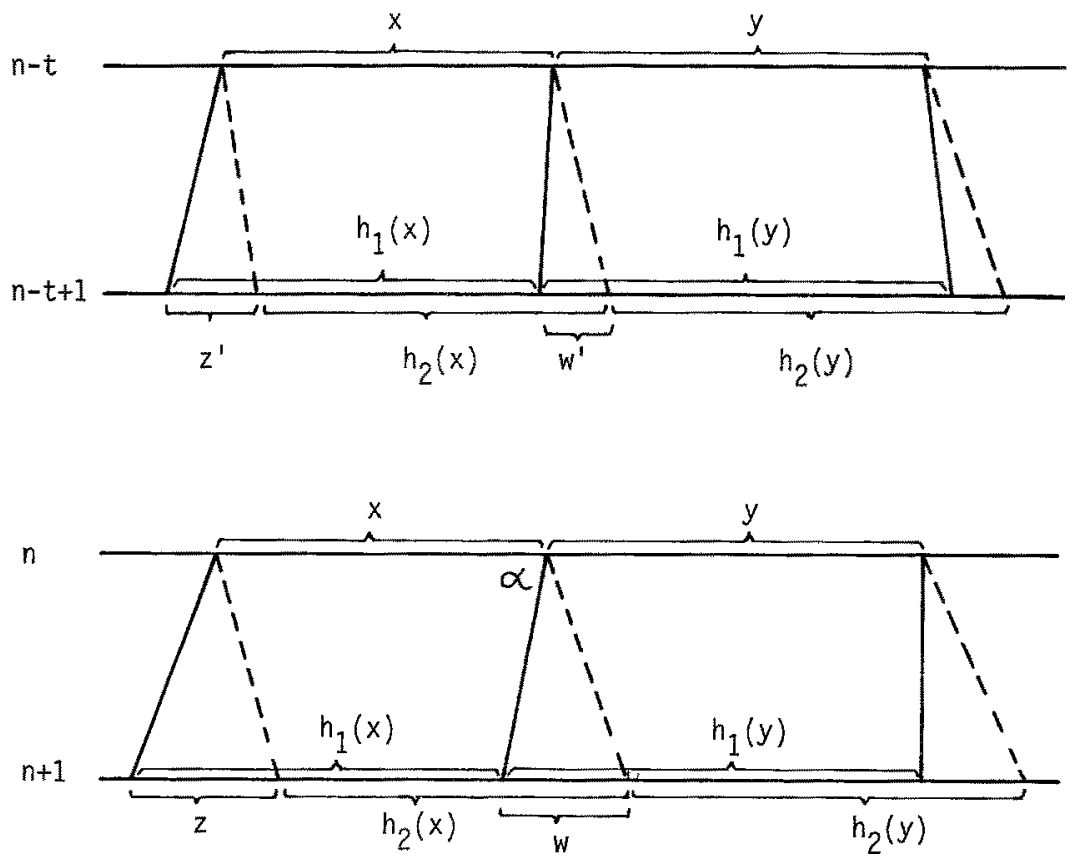


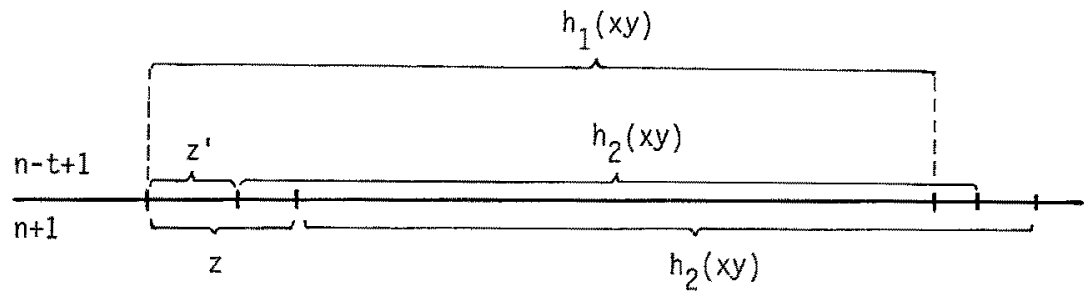

Fig. 3

So if we compare levels $n-t+1$ and $n+1$, as shown on Fig. 3., by matching the common substring $h_{1}(x y)$ we see that $h_{2}(x y) w_{1}=w_{2} h_{2}(x y)$ for some "very short" strings $w_{1}$ and $w_{2}$. Therefore the string $h_{2}(x y)$ is periodical with period much shorter than both $x$ and $y$. Therefore there exists $r$ such that $r$ is a suffix of $x$ and prefix of $y$ as illustrated on Fig. 4.

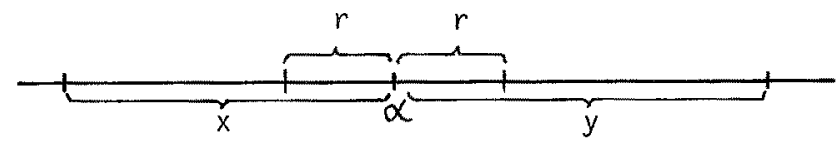

Fig. 4

So far we have used only the fact $\beta(u)$ is strictly maximal up to level $n$ (condition (i)), but not yet the conditions (ii) and (iii) implied by Assumption 1. Now we will exploit them by considering two cases:

Case I. Let $\beta(r)=0$. A suffix of $x$ is also a suffix of $u$ so $u=\bar{w} r$ for some $\bar{w}$ in $\Sigma^{*}$ and therefore $\beta(u)=\beta(\bar{w})$ which is in contradiction with condition (iii) implied by Assumption 1 .

Case II. Let $B(r)>0$. Since $r$ is also a prefix of $y$ we can write $h_{1}^{n}(\sigma)=\overline{u r}^{2} \vec{v}$ for 
some $\bar{u}, \bar{v}$ in $\Sigma^{*}$ such that $\bar{u} r=u$ and $r \bar{v}=v$. Since $\beta(r)>0$, clearly, either $B(\bar{u})>\beta(u)$ or $\beta\left(\bar{u} r^{2}\right)>\beta(u)$ which is in contradiction with condition (ii) implied by Assumption 1 .

\section{Subalphabets, remainder systems and induced systems.}

Given a DOL system $G=(\Sigma, h ; \sigma)$, a nonempty set II properly contained in $\Sigma_{c}$ is called a subalphabet if $h(a) \in \Pi^{*}$ for each $a \in \Pi$. Let $\Omega=\Sigma-\Pi$. If $G$ is an $2 r$-system we will also use $\Omega_{c}$ for $\Sigma_{c}-\pi$. For every $z \in \Sigma^{*}$ we denote by $z^{\Omega}$ the string $z$ with all symbols from II omitted, thus $z^{\Omega} \in \Omega^{*}$. We define $G^{\Omega}$ as $\left\langle\Omega, h^{\Omega}, \sigma^{\Omega}>\right.$ where $h^{\Omega}(x)=(h(x))^{\Omega}$ for $x \in \Omega$. If for a sequence $s=s_{1}, s_{2}, \ldots$ we write $s^{\Omega}=s_{1}^{\Omega}, s_{2}^{\Omega}, \ldots$, then obyiously

(4) $\quad(s(G))^{\Omega}=S\left(G^{\Omega}\right)$

where $s(G)$ is the sequence generated by DOL system $G$.

Given two DOL systems $G_{1}, G_{2}$, II is called their common subalphabet if $\pi$ is a subalphabet of $G_{i}$ for $i=1,2$. From (4) we get immediately that if $G_{1}, G_{2}$ are equivalent and have a common subalphabet II then also the remainder systems $G_{1}^{\Omega}$ and $G_{2}^{\Omega}$ are equivalent. It is also obvious that if $G$ is normal, so is $G^{\Omega}$.

Lemma 2 Let $G_{i}=\left\langle\Sigma, h_{i}, 0\right\rangle, i=1,2$ be two normal propagating equivalent DOL systems. Then $G_{1}$ and $G_{2}$ have a common subalphabet $I$, or the composite homomorphism $h_{1} h_{2}$ is Ir-simple.

Proof Outline (for details see [2], Lemma 5). Assume that $G_{1}$ and $G_{2}$ have no common subalphabet. First we show that all symbols in $\Sigma_{c}$ occur in infinitely many strings of $L\left(G_{1}\right)$. Let $\Gamma$ be the set of symbols which occur only finitely many times. Since $G_{1}$ and $G_{2}$ are equivalent clearly either $\Sigma-\Gamma$ is a subalphabet or $\Gamma$ is empty.

Now, write $a \vec{j} b$ for $a, b \in \Sigma_{c}$ and $i=1,2$ if $b \in m_{i}(a)$, i.e. a directly derives $b$ in $G_{i}$. Let $\Rightarrow=\vec{I} \quad \cup \quad \overrightarrow{2}$ and $\Rightarrow^{*}$ be the transitive and reflexive closure of $\Rightarrow$. Finally, let $\tilde{m}(a)=\left\{b \stackrel{1}{\epsilon} \Sigma_{c}::^{2} a * b\right\}$. Obvious $7 y, m_{j}(\tilde{m}(a)) \subseteq \tilde{m}(a)$ for $j=1,2$, so $\tilde{m}(a)=\Sigma_{c}$ since otherwise $\tilde{m}(a)$ would be a subalphabet. This means that $a \Rightarrow b$ for any $a, b \in \Sigma_{c}$. In more detail we can write $a \underset{i_{1}}{c_{1}}{\overrightarrow{i_{2}}}_{2} c_{2} \overrightarrow{i_{3}} \ldots \ldots \underset{i_{n-1}}{\longrightarrow} c_{n} \overrightarrow{i_{n}} b$, for $n \geq 0$, $i_{j} \in\{1,2\}$ and $c_{i} \in \Sigma_{c}$. Because $G_{1}$ and $G_{2}$ are propagating and have no common subalphabet we can choose $i_{1}=1$ and $i_{n}=2$. From the normality it also follows that we can "abbreviate" several successive transfers by either $\overrightarrow{1}$ or $\overrightarrow{2}$ into one such transfer, so we get a $\vec{i} \quad d_{1} \overrightarrow{2} \quad d_{2} \vec{l} \cdots \cdot \vec{l} d_{m} \overrightarrow{2} \quad b$ for some $0 \leq m \leq n$ and $d_{i} \in \Sigma_{c}$. Therefore $a \underset{1,2}{\longrightarrow} d_{2} \underset{1,2}{\longrightarrow} d_{4} \cdots \underset{1,2}{\longrightarrow}$ b where $x \underset{1,2}{\longrightarrow}$ y means that $y \in m_{1,2}(x)$.

We have shown that for any $a, b \in \Sigma_{c}$ we have $a \overrightarrow{1,2}^{*} b$. That means that the growth matrix $M_{1,2}$ associated with $h_{1,2}$ is in terminotogy of [4] irreducible. Now, we need only to show a stronger property, namely that a system is $2 r-s$ imple if its growth matrix restricted to $\Sigma_{c}$ is primitive (see [4]). From results in [4] it follows that 
if the growth matrix is not primitive then there exist $q>I$ and a partition $P$ of $\Sigma_{C}$ with $q$ classes such that for every $a, b \in \Sigma_{c}$, if $a \in m_{1,2}^{q}(b)$, then $a$ and $b$ belong to the same class of $P$. We use again the normality of $G_{1}$ and $G_{2}$ to show that if $b \Rightarrow a$ then $a$ and $b$ must belong to the same class of $P$. Since $a \Rightarrow * b$ for any $a, b$ in $\Sigma_{c}$ there cannot exist a partition with at least two classes and that completes the proof that $h_{1} h_{2}$ is $z$-simple.

Definition 4 Given $G=\left\langle\sum h, \sigma\right\rangle$. A subalphabet $\pi \subsetneq \sum$ is called zimited if there is a constant $k$ such that for every substring $u \in I^{*}$ of $L(G)$ we have $|u|<k$. Note that $I$ is limited with respect to every DOL system equivalent to $G$.

Lerma 3 Let $G_{1}, G_{2}$ be two equivalent systems, with a common subalphabet $I$. If II is limited and if the pair $\left(G_{1}^{\Omega}, G_{2}^{\Omega}\right)$ has a bounded balance, then the pair $\left(G_{1}, G_{2}\right)$ has bounded balance.

Proof Let the balance of $\left(G_{1}^{\Omega}, G_{2}^{\Omega}\right)$ be $c$ and let $k$ be such that $|u| \leq k$ for all $G_{1}-$ substrings $u$ from $\Pi^{*}$. Then the balance of the pair $\left(G_{1}, G_{2}\right)$ is clearly smaller or equal to $(c+1) k+c$.

Definition 5 Let $G_{1}, G_{2}$ be a pair of DOL systems, $G_{i}=\left(\Sigma, h_{j}, \sigma\right)$. Given $1=i_{1} i_{2} \ldots i_{n}$ with $n \geq 1$ and $i_{1}, \ldots, i_{n} \in\{1,2\}$, the set $S=\left\{G_{1}^{j}, G_{2}^{j}: 0 \leq j \leq n\right\}$ of pairs of $D O L$ systems is called i-combination of $\left(G_{1}, G_{2}\right)$ where $G_{i}^{j}=\left(\Sigma, h_{j}, \sigma_{i j}\right)$, for $i=1,2, j=0$, $\ldots, n, \bar{h}_{1}=h_{1} h_{i_{1}} h_{i_{2}} \ldots h_{i_{n}}, \bar{h}_{2}=h_{2} h_{i_{1}} h_{i_{2}} \ldots h_{i_{n}}, \sigma_{i, 0}=\sigma$ and $\sigma_{i, j}=h_{i j} \ldots h_{i_{n}}(\sigma)$ for $i=1,2$ and $j=1, \ldots, n$. Finally, we reduce each system $G_{j}^{j}$, if necessary.

Instead of 1-combination we will say just combination. If $2=(21)^{\mathrm{k}}$ for the minimal $k \geq 0$ such that each $G_{i}^{j}$ is normal we call the 1 -combination the normal combination of $\left(G_{1}, G_{2}\right)$. We show that for normal systems $G_{1}, G_{2}$ such $k$ always exists. We find $k$ so that $m_{21}^{k}(a)=m_{21}^{k s}(a)$ for all a $\in \sum$ and $s=1,2, \ldots$ (see [2], Lemma 2.). Therefore also $m_{i}\left(m_{21}^{k}(a)\right)=m_{i}\left(m_{21}^{k s}(a)\right)$ for $i=1,2$, and $s=1,2, \ldots$. Now, to show that the homomorphisms of the normal combination satisfy condition (3) of normality we note that

$$
m_{2}^{k_{1}} m_{1}^{k_{2}} \ldots m_{2}^{k_{2 n-1}} m_{1}^{k_{2 n}}(a)=m_{21}^{n}(a)
$$

for each aEs, $n \geq 1$ and arbitrary $k_{1}, \ldots, k_{2 n} \geq 1$; since, because of normality of $G_{1}$ and $G_{2}$, the repetitions of the same homomorphisms are irrelevant. Specifically,

$$
\begin{aligned}
& {\left[m_{2} m_{21}^{k}\right]^{s}(a)=m_{21}^{k s}(a)=m_{21}^{k}(a)=m_{2} m_{21}^{k}(a)} \\
& {\left[\begin{array}{ll}
m_{1} & m_{21}^{k}
\end{array}\right]^{s}(a)=m_{1} m_{21}^{k s}(a)=m_{1} m_{21}^{k}(a)}
\end{aligned}
$$

and

for each $a \in \Sigma$ and $s \geq 1$, which shows that the systems of a normal combination satisfy condition (3) of normality. 
We will say that the set $S$ has bounded balance if each pair $\left(G_{1}^{j}, G_{2}^{j}\right) \in S$ has bounded balance.

Lemma 4 Let $\left(G_{1}, G_{2}\right)$ be a pair of DOL systems. Let $S$ be their 1 -combination for some $\imath \in\{1,2\}^{+}$. Then

$$
G_{1}, G_{2} \text { are equivalent iff for all }\left(G_{1}^{j}, G_{2}^{j}\right) \in S, G_{1}^{j}, G_{2}^{j} \text { are equivalent. }
$$

Let $G_{1}$ and $G_{2}$ be equivalent. Then $\left(G_{1}, G_{2}\right)$ has bounded balance iff their 2 -combination $S$ has bounded balance.

Proof Part (i) can be proved analogically as Theorem 1. Now, let $k=|l|$ and assume that $\left(G_{1}^{j}, G_{2}^{j}\right)$ has bounded balance and let $w$ be a $G_{1}$-prefix, say, $w w^{\prime}=h_{l}^{n}(\sigma)$ for some $n \geq 0$ and some $W^{\prime} \in \Sigma^{*}$. When proving that the balance is bounded on a set of strings we may neglect finitely many strings, so, let $n \geq k$. Let ${ }^{2}=i_{1} i_{2} \ldots i_{k}$ and $h=h_{i_{1}} h_{i_{2}} \ldots h_{i_{k}}$. Let ua with $u \in \Sigma^{*}$, aE be a prefix of $h_{1}^{n-k}(\sigma)$ such that $h(u)$ is a prefix of $w$, but $w$ is a proper prefix of $h(u a)$. (Such ua exists if $w$ is a proper prefix, but if $w$ is the whole string $h_{1}^{n}(\sigma)$ then $\beta(w)=0$, so again we may ignore this), i.e. $h(u) x=w$ for some $x \in \Sigma^{*}$, and $x$ is a prefix of $h(a)$, from which $|x| \leq H^{k}$ and $B(x) \leq B H^{k}$, i.e. $B(w) \leq \beta(h(u))+B H^{k}$, where $H=\max _{a \in \Sigma}\left|h_{1}(a), h_{2}(a)\right|$ and $B=\max _{a \in \Sigma} \beta(a)$. The boundedness of $\beta(w)$ follows from the fact that $\beta(h(u))=|| \hbar_{1}(u)|-| h_{2}(u)||=\beta_{j}(u)$, where we denoted by $\beta_{j}$ the balance in $\left(G_{1}^{j}, G_{2}^{j}\right)$ which is bounded, and $j$ is chosen so that $w$ is a $G_{1}^{j}$-prefix.

The converse, namely, that if $\left(G_{1}, \hat{W}_{2}\right)$ has bounded balance so has each $\left(G_{1}^{j}, G_{2}^{j}\right)$ is obvious, and is not in fact needed in our proofs.

Definition 6 Let $G=(\Sigma, h, \sigma)$ be a DoL system and let $\Pi \subset \Sigma$ be a subalphabet, and assume that $h^{\Omega}$ is propagating. For every avb $\in \Omega \pi *_{\Omega}$ we define an induced system $G^{a v b}=\left(\Sigma^{a}+\Pi^{\prime}+{ }^{b} \Sigma, \bar{h}, \bar{a} v \bar{b}\right)$ as follows.

For $a \in \Omega$, let $h(a)=x c v$, where $c \in \Omega, V \in I^{*}$. (Note that such decomposition is possible because $h^{\Omega}$ is propagating, and is obviously unique.) We denote $z(a)=c, z^{\prime}(a)$ $=v$. Similarly, writing $h(a)=v^{\prime} c^{\prime} y$, where $c^{\prime} \in \Omega, v^{\prime} \in \Pi^{*}$, we define $r(a)=c^{\prime}$, $r^{\prime}(a)=v^{\prime}$.

We define $\Sigma^{a}=\left\{\bar{c}\right.$ : there is $n \geq 0$ and a sequence $c_{0}=a, c_{1}, \ldots, c_{n-1}, c_{n}=c, c_{j} \in \Omega$ such that $\left.c_{j}=z\left(c_{j^{1}}\right), j=1,2, \ldots, n\right\}$, where $\bar{c}$ is one new symbol for each $c \in \Omega$. Similarly, we define $b_{\Sigma}$ starting with $c_{0}=b$ and using $r$ instead of $z: b_{\Sigma}=\{\overline{\bar{c}}$ : there is $m \geq 0$ and a sequence $c_{0}=b, c_{1}, \ldots, c_{m}=c, c_{j} \in \Omega$ and $c_{j}=r\left(c_{j-1}\right)$ for $j=1,2$, $\ldots, m\}$, and where $\overline{\bar{c}}$ is another new symbol, one for each $c \in \Omega$. Let $\hat{h}(\bar{a})=\overline{z(a)} z^{\prime}(a)$ for $a \in \Omega, \bar{h}(\overline{\bar{a}})=r^{\prime}(a) \overline{\bar{r}(a)}$ for $a \in \Omega, \bar{h}(d)=h(d)$ for $d \in \Pi$. Finaliy, $\Pi^{\prime}$ is the subset of $\pi$ of symbols actually used when the homomorphism $\hat{h}$ is repeatedly applied to $v$. That completes the definition of $G^{a v b}$. When starting with $G_{1}$ or $G_{2}$ we will, as 
usual, talk about $\bar{h}_{1}, \bar{h}_{2}, G_{1}^{a v b}$, and $G_{2}^{a v b}$.

Lemma 5 Let $G_{1}, G_{2}$ be two equivalent DOL systems with a common subalphabet $I$. Assume both $h_{1}$ and $h_{1}^{\Omega}$ are propagating and there exists a constant $k$ such that for every $G_{1}$ prefix of the form $x a v$, where $a \in \Omega, x \in \Sigma^{*}$, and $v \in I^{*}$ we have

$$
\text { if }|v|>k \text {, then } h_{1}^{\Omega}(x a)=h_{2}^{\Omega}(x a) \text {. }
$$

Then for every avb $\in \Omega \Omega_{\Omega},|v|>k$, avb a substring of $L\left(G_{1}\right)$ the systems $G_{1}^{a v b}$, $G_{2}^{a v b}$ are equivalent.

Proof As avb is a $G_{1}$-substring, we can write xavby $=h_{1}^{j}(\sigma)$ for some $x, y \in \Sigma *$ and some $j \geq 0$. From (5) we have $h_{1}(x a)=x^{\prime} z_{1}(a) z_{1}^{\prime}(a), h_{2}(x a)=x^{\prime} z_{2}(a) z_{2}^{\prime}(a)$, where $z_{1}, z_{1}^{\prime}$ and $z_{2}, z_{2}^{\prime}$ are functions from definition 6 based here on $h_{1}$ and $h_{2}$. Similarly, $h_{i}(x a v b)=x^{\prime} \tau_{j}(a) z_{i}^{\prime}(a) h_{i}(v) r_{j}^{\prime}(b) r_{i}(b) x_{j}^{\prime \prime}$, for some $x^{\prime}, x_{i}^{\prime \prime} \in \Sigma^{*}, i=1,2$. Strings $h_{1}(x a), h_{2}(x a)$ and $h_{1}(x a v b), h_{2}(x a v b)$ are prefixes of the same string $h_{1}^{j+1}(\sigma)=h_{2}^{j+1}(\sigma)$, so $z_{1}(a)=z_{2}(a) \in \Omega ; z_{1}^{\prime}(a) h_{1}(v) r_{1}^{\prime}(b)$ and $z_{2}^{\prime}(a) h_{2}(v) r_{2}^{\prime}(b) \in \Pi^{*}$, but they are equal as the next symbol $r_{1}(b)=r_{2}(b) \in \Omega$. That is, $|v|>k$ implies (through $h_{1}^{\Omega}(x a)=h_{2}^{\Omega}(x a)$ ) that $\hat{h}_{1}(\bar{a} v \bar{b})=\hat{h}_{2}(\bar{a} v \overline{\bar{b}})$. As $h_{1}$ - and thus $\hat{h}_{1}$ - are propagating, also $\left|h_{1}(v)\right| \geq|v|>k$. This proves that $\mathrm{G}_{1}^{\mathrm{avb}}, \mathrm{G}_{2}^{\mathrm{avb}}$ are equivaient.

口

\section{The main theorem}

Theorem 4 Every pair of normal equivalent DOL systems has bounded balance.

Proof Outline (for details see [2], Theorem 3.)

Let $G_{i}=\left(\Sigma, h_{j}, \sigma\right)$ for $i=1,2$; and let $r$ be the order of $G_{1}$ (same as $G_{2}$ ), i. e. $r=\left|\Sigma_{c}\right|$. The proof is by the induction on $r$. It is easy to verify that the assertion holds for systems with one-letter core $\left(\left.\right|_{c} !=1\right)$ which serves as base, $r=1$, of the induction.

The induction hypothesis states that the assertion holds for systems of order smaller than $r \geq 1$. To prove the induction step let us consider two normal equivalent systems of order $r$, i.e. $\left|\Sigma_{c}\right|=r \geq 2$.

Case I: Assume that $h_{1}(a)=h_{2}(a)=\varepsilon$ for some $a \in \Sigma_{c}$. Then $\pi=\{a\}$ is a common subalphabet. Let $\Omega=\Sigma-I$. Since $G_{1}$ and $G_{2}$ are equivalent also $G_{1}^{\Omega}$ and $G_{2}^{\Omega}$ are equivalent and since $\left|\Omega_{c}\right|<\left|\Sigma_{c}\right|$ the pair $\left(G_{1}^{\Omega}, G_{2}^{\Omega}\right)$ has bounded balance by the induction hypothesis. Subalphabet $I$ is clearly limited and therefore the pair $\left(G_{1}, G_{2}\right)$ has bounded balance by Lemma 3 .

Case II: Assume that $h_{1}(a)=\varepsilon$ for some $a \in \Sigma_{c}$ but not necessarily $h_{2}(a)=\varepsilon$. Consider the normal combination of $\left(G_{1}, G_{2}\right)$. Clearly, we have $\bar{h}_{1}(a)=\varepsilon, \bar{h}_{2}(a)=\varepsilon$, so by case $I$, $\left(G_{1}^{i}, G_{2}^{i}\right)$ has bounded balance for $i=1,2$ and so has $\left(G_{1}, G_{2}\right)$ by Lemma 4 .

Case III. This is the most difficult case and we will give only a very rough outline 
of the proof, which omits many difficuit details (see [2], Lemma 9. clairo 1, cases $A-D$ and Subcases III $A-$ III D).

We may now assume that both $G_{1}$ and $G_{2}$ are propagating. By Lemma 2 either the combination of $\left(G_{1}, G_{2}\right)$ is simple (this implies, using Theorem 3 and Lemma 4 , that $\left(G_{1}, G_{2}\right.$ ) has bounded balance), or there is a common subalphabet $\Pi$. Let $\Omega=\Sigma-\pi$ and $\Omega_{C}=\Sigma_{C}-\Pi$.

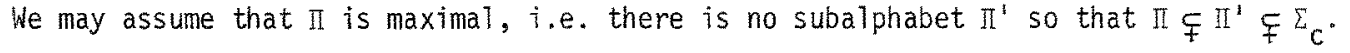
Since $G_{1}$ and $G_{2}$ are equivalent the remainder systems $G_{1}$ and $G_{2}$ are also equivalent, and since they are of order smaller than $r$, and normal, the pair $\left(G_{1}, G_{2}\right)$ has bounded balance by the induction hypothesis.

Now, we consider any $G_{1}$-prefix $w$. If all substrings of $w$ in $I^{*}$ are "short", we bound $\beta(w)$ easily. To take care of the $G_{1}$-prefixes with long substrings in $\mathbb{I}^{+}$we first show that there is only a finite number of induced systems of $G_{1}$ or $G_{2}$ generating all such "long" substrings in $\pi^{+}$with "sides" from $\Omega$.

The next difficult step is to show that for every $G_{1}$-prefix uv with $u \in \Sigma * \Omega$ and $v \in \mathbb{I}^{+}$ where $v$ is "long" we have $\beta(u)=0$, i.e. that the balance is zero at every point where a "long" substring in $\pi^{+}$starts. Therefore, using Lemma 5 , we show that the corresponding induced systems $G_{1}^{a v b}$ and $G_{2}^{a v b}$ for each "long" $v$ in $\mathbb{\pi}^{+}$are equivaient. Since $G_{1}^{a v b}$ and $G_{2}^{a v b}$ are of order less than $r$ they have bounded balance by the induction hypothesis.

Now, we are finally in the position to show that the balance is bounded also on $G_{1}$ prefixes with "long" substrings in $\pi^{+}$. We can write each such $G_{1}$-prefix $w$ in the form $w=x y z$ where $x \in \Sigma^{*} \Omega, y \in \mathbb{I}^{+}, z \in \Sigma^{*}$ where $y$ is the last "long" substring in $\mathbb{I}^{+}$from left, i.e. $z$ does not contain any "long" substring in $\pi^{+}$(possibly $z=\varepsilon$ ). It is essential that we already know that $B(x)=0$. Let $H=\max _{i=1,2}\left(\max _{a \in \Sigma} h_{j}(a)\right), c$ be the bound on

balance of $G_{1}, G_{2}$ and $C_{\pi}$ be the maximum of bounds on balance of the finitely many pairs of induced systems. From $\beta^{\Omega}(x)=0$, it follows that $\beta(x) \leq H$. Also $B(y) \leq c_{\pi}+2 H$ and finally $B(z) \leq C\left(H^{C}+1\right) H$. So together we have $B(w) \leq B(x)+B(y)+B(z) \leq H+C_{\pi}+2 H$ $+c\left(H^{C}+1\right) H$ and therefore the balance of the pair $G_{1}, G_{2}$ is bounded.

\section{Equivalent systems with bounded balance}

Now, we show that when we consider only DOL systems for which equivalence implies bounded balance, we can design a decision procedure for equivalence. We conjecture that this implication holds for all DoL systems, however in view of Theorem 1 it is sufficient that it holds for normal systems as proved in section 5.

Theorem 5 Let $F$ be a family of DOL systems with the property: (*) If $G_{1}$ and $G_{2}$ are two equivalent systems in $F$, then the pair $\left(G_{1}, G_{2}\right)$ has bounded balance. Then the sequence equivalence problem for $F$ is decidable.

Proof (see also [1], Theorem 2.1). Clearly, we can restrict ourselves to a pair of 
reduced DOL systems from $F$ with identical alphabets and identical axioms. We will exhibit two semidecision procedures, one for nonequivalence and the other for equivalence.

The semidecision procedure for nonequivalence is trivial, we compute $h_{1}^{n}(\sigma)$ and $h_{2}^{n}(\sigma)$ for $n=0,1,2, \ldots$ and stop with answer "nonequivalent" if $h_{1}^{n}(\sigma) \neq h_{2}^{n}(\sigma)$ for some $n$.

our semiprocedure for equivalence is based on the condition (*) for $F$, namely that a pair of equivalent systems from $F$ has bounded balance.

Clearly, $h_{1}^{n}(\sigma)=h_{2}^{n}(\sigma)$ for $n \geq 0$ iff $h_{1}^{n}(\sigma)=h_{2}\left(h_{1}^{n-1}(\sigma)\right)$ for $n>0$ iff $h_{1}(w)=h_{2}(w)$ for each $w$ in $L\left(G_{1}\right) . L\left(G_{1}\right)$ is a $D O L$ language and therefore also an EOL-language [9].

Now we design a semiprocedure which will check successively for $k=1,2, \ldots$ whether the pair $\left(G_{1}, G_{2}\right)$ has k-bounded balance and whether $G_{1}$ and $G_{2}$ are sequence equivalent. We already know that to check the sequence equivalence it is enough to check whether $h_{1}(w)=h_{2}(w)$ for each $w \in L\left(G_{1}\right)$. The checking of these two properties for a particular $k$ is done as follows:

Let $M_{k}$ be a deterministic g.s.m [10] with a "buffer" of length $k$ in its finite control which for any input string $w$ in $\Sigma$ attempts to check (from left to right when reading w) whether $h_{1}(w)=h_{2}(w)$. It is obviously possible to do this if $G_{1}$ and $G_{2}$ have kbounded balance since we have available a "buffer" of length $k$ (i.e. a buffer able to contain $k$ symbols from $\Sigma$ ). Given input $w$, our g.s.m. $M_{k}$ will produce its output as follows:

(i) If the buffer of $M_{k}$ does not overflow and $h_{1}(w)=h_{2}(w)$, then no output is produced ( $M_{k}$ goes into a non-accepting state).

(ii) Otherwise, $\left(M_{k}\right.$ finds that $h_{1}(v) \neq h_{2}(v)$ for some prefix $v$ of $w$ or the buffer overflows), $M_{k}$ stops in an accepting state and therefore produces some output (what output is produced is irrelevant).

Clearly, $T_{k}\left(L\left(G_{1}\right)\right)=\emptyset$ iff the pair $\left(G_{1}, G_{2}\right)$ has $k$-bounded balance and $h_{1}(w)=h_{2}(w)$ for all $w \in L\left(G_{1}\right)$. Since EOL-systems are effectively closed under g.s.m. mappings [9] we can construct an EOL-system $S_{k}$ such that $L\left(S_{k}\right)=T_{k}\left(G_{1}\right)$. Finally, it is recursively decidable $[3,9]$ whether the EOL-language $L\left(S_{k}\right)$ is empty. Therefore, simply enumerate $S_{1}, S_{2}, \ldots$ and test each $S_{k}$ for $L\left(S_{k}\right)=\emptyset$. The systems $G_{1}$ and $G_{2}$ are equivalent iff there is $k$ so that $L\left(S_{k}\right)=0$.

Our semiprocedure must eventually stop if $G_{1} \equiv G_{2}$ since, because condition (*) for $F$, there exists $c>0$ so that $G_{1}$ and $G_{2}$ have $c$-bounded balance.

Corollary 1 The sequence equivalence problem for DoL systems is decidable.

Proof Theorem 4 shows that the family of normal systems satisfies the condition (*) of Theorem 5, therefore, by Theorem 5, the sequence equivalence problem is decidable for this family. Thus, by Theorem 1, the sequence equivalence problem is decidable for all DOL systems. 
Corollary 2 The language equivalence problem for DOL systems is decidable, i.e. given two DOL systems $G_{1}, G_{2}$, it is decidable whether $L\left(G_{1}\right)=L\left(G_{2}\right)$.

Proof By Corollary 1 and Corollary 4.6 in [6].

$\square$

\section{References}

1. Culik II, K., On the decidabitity of the sequence equivalence problem for DOL-systems, Theoretical Computer Science 3, 75-84 (1977).

2. Culik II, K. and Fris, 1., The decidability of the equivalence problem for DOLsystems, Inf. and Control, in print, also Res. Rep. CS-76-25, Dept. of Computer Science, University of Waterloo (1976).

3. Culik II, K., On some families of languages related to developmental systems, Inter. J. of Computer Math. 4, Section A, 31-42 (1974).

4. Gantmacher, F. R., The Theory of Matrices, vol. 2, Chelsea, New York (1960).

5. Lindenmayer, A., Developmental systems without cellular interaction, their languages and grammar, J. of Theoretical Biology 30, 455-484 (1971).

6. Nielsen, M., On the decidability of some equivalence problem for DOL-systems, Information and Control 25, 166-193 (1974).

7. Paz, A. and Salomaa, A., Integral sequential word functions and growth equivalence of Lindenmayer systems, Information and Control 23, 313-343 (1973).

8. Problem Book, Unusual Automata Theory, January 1972, Dept. of Computer Science, University of Aarhus Techn. rep. DAIMI PB-15, 14-26 (1973).

9. Rozenberg, G., Extensions of tabled OL-systems and languages, Inter.J. of Computer and Information Sciences, Vol. 2, 311-334 (1973).

10. Salomaa, A., Formal Languages, Academic Press, New York, (1973). 